\title{
BREDEKAMP, Horst, SCHNEIDER, Pablo, Visuelle Argumentationen. Die Mysterien der Repräsentation und die Berechenbarkeit der Welt
}

\section{Naïma Ghermani}

\section{(2) OpenEdition}

\section{Journals}

Édition électronique

URL : http://journals.openedition.org/ifha/658

DOI : $10.4000 /$ ifha.658

ISSN : 2198-8943

Éditeur

IFRA - Institut franco-allemand (sciences historiques et sociales)

Référence électronique

Naïma Ghermani, « BREDEKAMP, Horst, SCHNEIDER, Pablo, Visuelle Argumentationen. Die Mysterien der Repräsentation und die Berechenbarkeit der Welt », Revue de I'IFHA [En ligne], Date de recension, mis en ligne le 01 janvier 2006, consulté le 22 septembre 2020. URL : http://journals.openedition.org/ifha/658 ; DOI : https://doi.org/10.4000/ifha.658

Ce document a été généré automatiquement le 22 septembre 2020.

(CIFHA 


\title{
BREDEKAMP, Horst, SCHNEIDER, Pablo, Visuelle Argumentationen. Die Mysterien der Repräsentation und die Berechenbarkeit der Welt
}

\author{
Naïma Ghermani
}

$\mathrm{Si}$, au XVIIe s., le pouvoir politique et notamment le pouvoir royal en Europe a tenté une mainmise et un contrôle sur les sciences, notamment à travers la fondation d'académies, accordant aux découvertes une ultime légitimité, les sciences et leur exactitude pouvaient, à l'inverse, entrer en contradiction avec certains « mystères de l'État ", voire les remettre en cause. C'est ce conflit, qui éclate particulièrement pendant le Grand Siècle, qui est ici l'objet de cet ouvrage collectif. Entre histoire des sciences, histoire politique et Bildwissenschaft, l'ouvrage s'intéresse particulièrement à l'image, espace où selon les deux éditeurs, le conflit éclate, car elle est aussi bien utilisée par les défenseurs du mystère du souverain que par les scientifiques dans leur argumentation. Lieu du déploiement d'une représentation théologique de la monarchie en France comme en Angleterre, elle est aussi l'objet d'une réflexion sur les techniques géométriques et mathématiques de la représentation.

2 Ce premier aspect est l'objet d'articles plus spécifiquement attachés à l'histoire de la philosophie, comme celui de K. LEONHARD qui tente, à la lumière des théories de Descartes principalement, de faire émerger le concept d'espace au XVIIe s., ou à l'histoire des sciences, comme celui S. BOGEN consacré aux représentations de machines et aux diagrammes qui offrent au XVIIe s. de véritables compendia mathématiques aux princes, tout en mettant au point un nouvel art de voir. Ph. VON HILGERS se penche, dans un article parfois trop abstrait, sur l'importance prise au XVIIe s. par les traités sur les jeux et leur stratégie, qu'il relie, à juste titre, à la réflexion de Leibniz sur le jeu comme ars characteristica et aux formes mathématiques d'organisation et de classement, tels les feuilles de calcul, les images géométriques et les diagrammes. L'auteur montre comment ces figures ou les Spielflächen 
entretiennent des relations étroites avec les traités de guerre et avec une conception du pouvoir comme un jeu, dans un État qui se technicise de plus en plus. La cartographie issue, elle aussi, d'une combinaison d'images, de chiffres et d'écriture peut devenir également productrice d'une conception du pouvoir et de son histoire. C'est ce que tente de montrer l'article de Wl. VELMINSKI en s'appuyant notamment sur la double et surprenante association de la cartographie de Saint-Pétersbourg et de sa présence chez Vermeer. A. FISCHEL poursuit dans cette veine analogue en proposant une réflexion sur les images microscopiques du XVIIIe s., entre image d'ornementation et image scientifique.

3 Plus spécifiquement, la question de la perspective qui affleure dans de nombreux articles, illustre particulièrement bien cette problématique. P. SCHNEIDER se penche sur les représentations de l'infinité du pouvoir, particulièrement frappantes dans les images de Versailles comme forme symbolique de l'absolutisme, et venant s'opposer à la finitude de certaines représentations du roi comme personne. La construction perspective des jardins, insérés dans une architecture militaire de la place forte laisse entrevoir, comme l'explique la contribution originale de Ch. BAIER et U. REINISCH, une stratégie visuelle peu connue dans les fortifications qui, au XVIIe s., laissent place dans leur enceinte à des « Lustgärten » établis savamment selon des règles mathématiques et qui traduisent le succès de l'ordre militaire et civil comme aune d'une société centralisée. C'est encore la perspective qui offre une nouvelle modalité d'expression symbolique du pouvoir du souverain et de sa dynastie dans les nouveaux ouvrages de généalogie produits dans la seconde moitié du XVIIe s., qui abandonnent la forme arborescente de présentation d'ancêtres - souvent imaginaires - pour la forme perspective aboutissant au dernier rejeton de la lignée. Ce changement de paradigme bien analysé par M. DISSELKAMP est à mettre en relation avec les transformations des techniques des arts de la mémoire et la revalorisation de certaines formes rhétoriques. Ces modifications des techniques cognitives apparaissent particulièrement dans le bel article de S. SIEGEL sur les représentations visuelles de Kunstkammern idéales. Le collectionnisme qui s'étend, en Europe, à toute une élite des cours et des villes, suscite la rédaction d'ouvrages spécialisés dans le classement parfait des objets disparates, empruntant à la rhétorique (la synecdoque comme principe de classement), au classement par affinité, à l'ordonnancement plus classique entre naturalia et artificialia, ou encore à un ordre guidé par la perspective.

4 La disposition de l'espace est ainsi au cœur du dispositif d'argumentation de l'image, et tout particulièrement pour les portraits de souverains. Ph. ZITZLSPERGER analyse ainsi finement l'échec du projet de buste de Louis XIV du Bernin et notamment de sa proposition d'un socle en forme de globe que dominerait la figure du souverain comparé, dans sa représentation, au Christ juge. Cette figuration de l'hybris du souverain par le sculpteur italien vient heurter l'idéal du portrait à la Cour qui préfère reléguer les symboles puissants à l'emblématique. L'efficacité du portrait du souverain repose encore sur sa spatialisation sur la toile : c'est ce que montre l'étude très précise et très documentée de C. BLÜMLE sur l'imposant portrait à cheval de Charles Ier d'Angleterre par Van Dyck, sous un arc de triomphe qui tranche avec les représentations traditionnelles du portrait royal en son Parlement et laisse entrevoir les tentations absolutistes du souverain. Le monarque absolu est encore au centre d'un article de B. SCHNEIDER sur une série de tapisseries des Gobelins, Histoire du Roy, un support souvent négligé par les historiens d'art et qui met en scène notamment la visite du roi aux Gobelins. Cette mise en abyme du travail de la tapisserie dans la tapisserie 
est réinsérée dans l'usage social de la tapisserie à la cour et est rattachée à sa fonction ostentatoire de consommation de luxe. L'auteur montre qu'elle donne lieu plus tard, à la fin du XVIIe et au XVIIIe s., à une représentation rationalisante des techniques de production et de son économie.

5 Cet ouvrage de qualité, issu d'un colloque organisé par le groupe de recherche « BildSchrift-Zahl », a le mérite de proposer une rencontre sinon inédite, du moins rare, entre histoire de l'art et histoire des sciences, une tendance, lancée notamment sous l'impulsion de H.B., qui permet d'ouvrir encore des champs nouveaux à l'interdisciplinarité.

6 Naïma GHERMANI (Université Pierre-Mendès-France - Grenoble II) 